\title{
Microstructure of Ultrasonic Welded Aluminum By Orientation Imaging Microscopy
}

\author{
E.A. Kenik* and R. Jahn** \\ *Oak Ridge National Laboratory, Metals and Ceramics Div., Oak Ridge, TN 37831-6064 \\ **Ford Research and Advanced Engineering, Dearborn, MI 48124
}

Ultrasonic spot welding (USW) of AA6111-T4 aluminum alloy has been achieved with attractive weld properties, even with surface contaminants (lubricant, oxide, etc.) present. USW provides a joining technique for aluminum and other light-weight materials in automotive and other transportation applications where weight-savings are an important consideration. Optical microscopy $(\mathrm{OM})$ has shown the localized formation and growth of solid-state welds. Though the grain structure of the base metal was apparent, OM failed to delineate a grain structure in the weld zones. The etched surface exhibited granularity at the micron level, but it was not clear if that reflected the grain structure. Orientation imaging microscopy (OIM) and electron backscatter diffraction (EBSD) were employed to characterize the developing microstructure of aluminum joined by USW.

Scanning electron microscopy (SEM) and OIM of electropolished cross-sections of ultrasonic welds were performed in an FEI XL30FEG SEM equipped with a TSL OIM system (generally performed at $10 \mathrm{kV}$ to improve spatial resolution of EBSD). As a result of the local deformation during USW, the image quality of EBSD patterns from the weld zone were lower than that for the base metal. Electropolishing produced a surface with minimal additional deformation from specimen preparation that yielded high quality EBSD diffraction patterns from the base metal and useable patterns from the weld zone. OIM maps were collected for 8-24 hours under computer control.

Figure 1(a) shows a secondary electron image of the weld interface in a weld (the image has been stretched vertically to correct for the $70^{\circ}$ tilt of the specimen and for easy comparison to the OIM maps). Slight surface relief from electropolishing highlights some grains in the base metal. Figure 1(b) shows an image quality (IQ) map at $0.5 \mu \mathrm{m}$ pixel spacing. Dark lines are often seen at grain and sub-grain boundaries where local lattice rotations result in poor image quality. An inverse pole figure (IPF) of the same area is shown in Fig. 1(c), where color indicates the orientation of a particular direction (e.g., the surface normal) in the 001/101/111 standard triangle. Some of the original coarse grain structure of the base metal is highlighted. Within each of these coarse grains the local rotations are less than $10^{\circ}$. As such the lines in the IQ map internal of the original grains are sub-grain boundaries. The size of grains decreases, whereas the misorientation across boundaries increases closer to the weld zone. There is a band of fine-grained material (arrowed) with diameters down to $\sim 1 \mu \mathrm{m}$. There is an abrupt change in the grain size near the upper edge of the weld zone.

Figure 2(a) is higher magnification inverse pole figure of the fine-grained region of a second weld taken at $0.2 \mu \mathrm{m}$ pixel spacing to better sample the fine grained region. This weld had shorter exposure to ultrasonic energy than the weld in Figure 1. However, the microstructures were similar with sub-grains in the base metal, fine grains in the weld zone and an abrupt change in grain size near one edge of the weld zone. Sub-micron grains are observed throughout this region. However, as these data were acquired at a pixel spacing of $0.2 \mu \mathrm{m}$, there is a size limit below which the indicated grains may be suspect. For an example of a 500-nm-diameter region, there are $\sim 7$ pixels and if all these pixels have the same orientation, it is reasonable for that region to be considered a grain. 
Smaller pixel spacing is required to correctly over-sample smaller grain sizes; though the ultimate spatial resolution of EBSD in a FEG-SEM (50-100 nm) also must be taken into account. Figure 2(b) gives the discrete pole figure for the region in Fig. 2(a) that shows the random orientation of the fine grains, indicating recrystallization of the heavily deformed weld zone. ${ }^{1}$

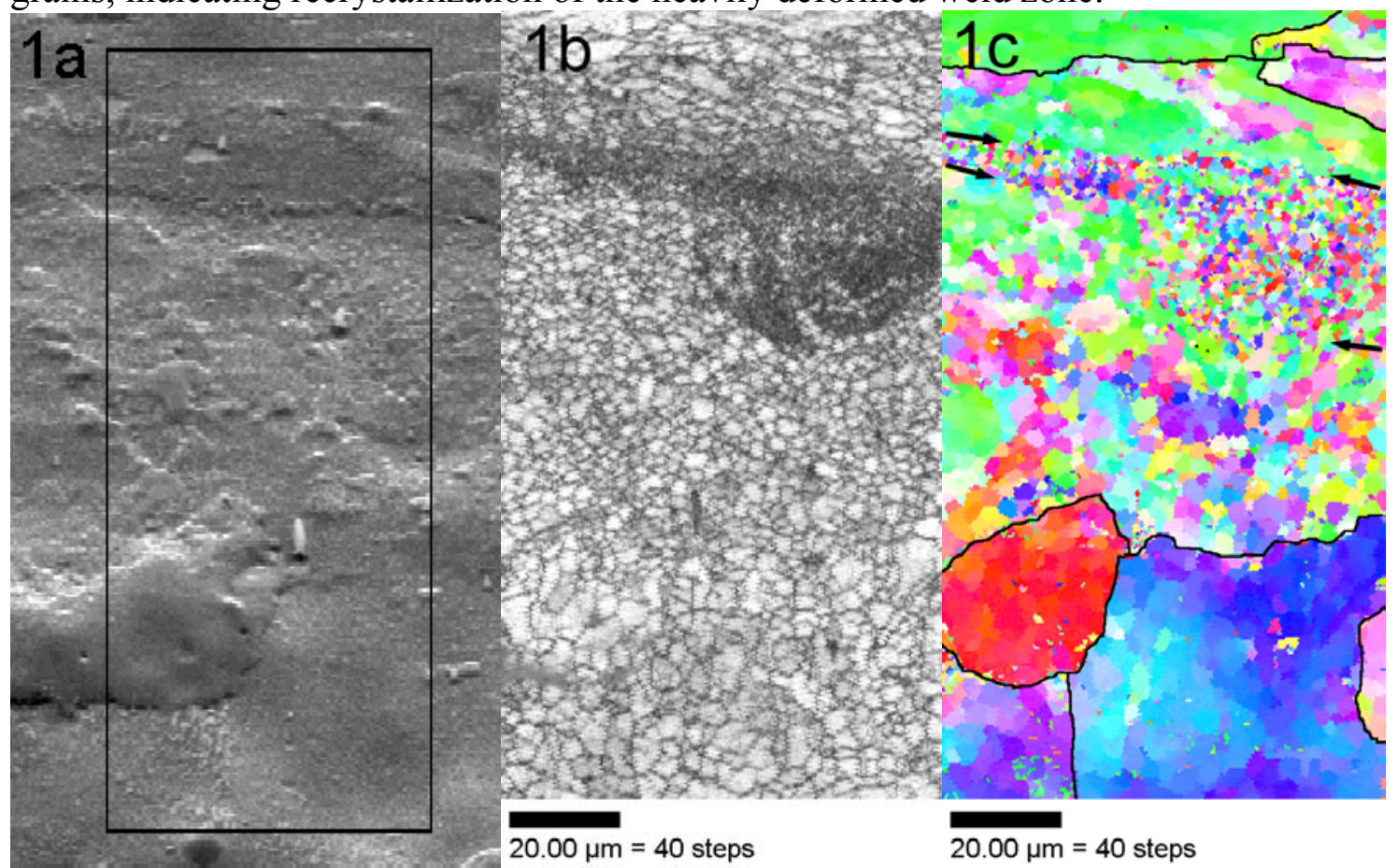

FIG. 1. (a) SEM image of weld zone. (b) Image quality map indicating boundaries. (c) Inverse pole figure showing the crystallographic orientation of the local surface normal.

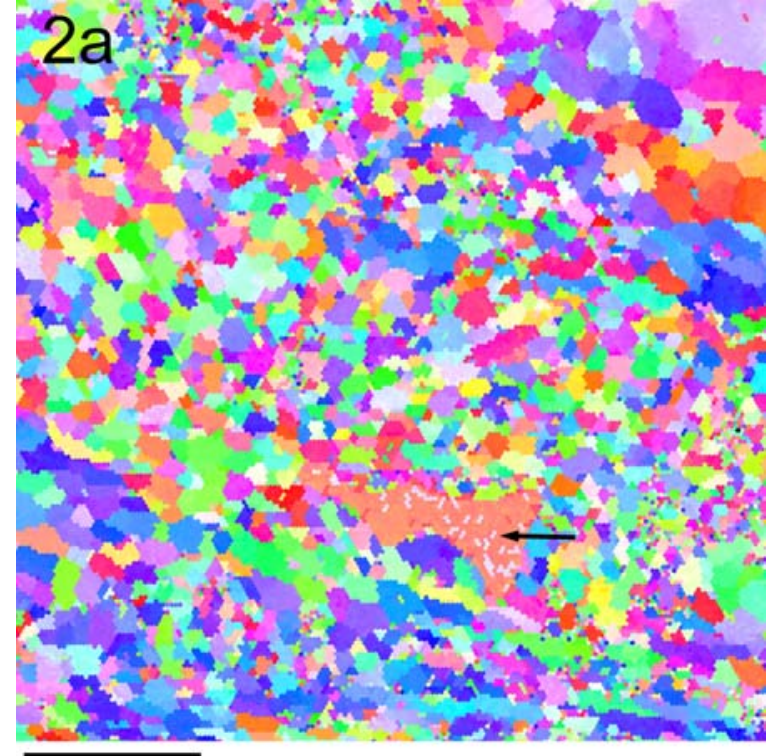

$8.00 \mu \mathrm{m}=40$ steps $\mathrm{IPF}[001]$

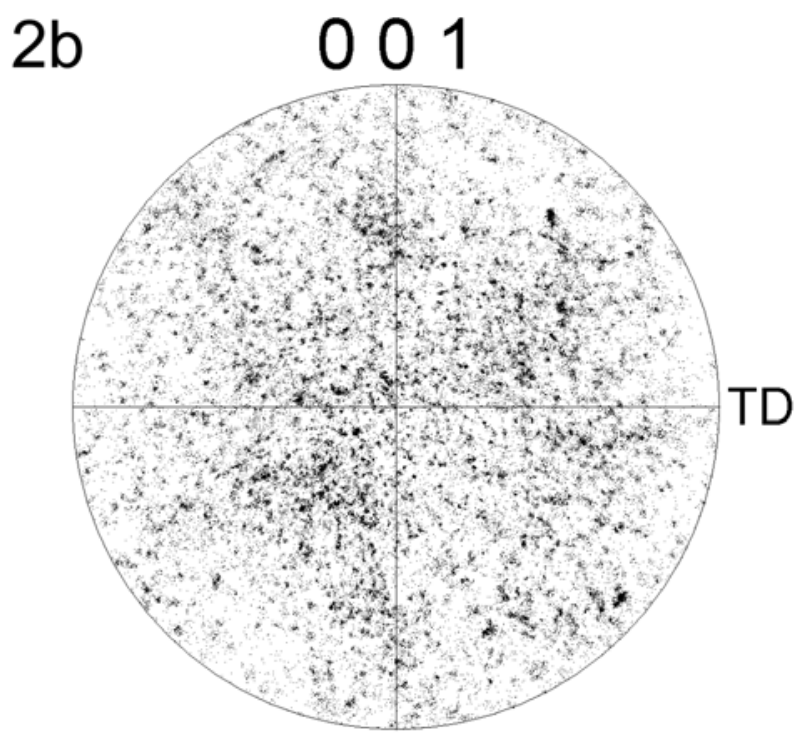

RD

FIG. 2. (a) IPF and (b) pole figure of fine-grained weld zone. Arrowed region is a coarse Al-Fe intermetallic phase.

(1) Research at the Oak Ridge National Laboratory SHaRE Collaborative Research Center was sponsored by the Assistant Secretary for Energy Efficiency and Renewable Energy, Industrial Technologies Program, Industrial Materials for the Future (MPLUS) and the Division of Materials Sciences and Engineering, U.S. Department of Energy, under contract DE-AC05-00OR22725 with UT-Battelle, LLC. 